\title{
is

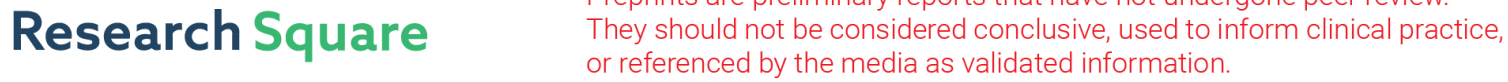 \\ Dyslipidemia and Its Associated Factors in Women of the Bandare-Kong Cohort Study
}

\section{Marzieh Nikparvar}

Cardiovascular Research Center, Hormozgan University of Medical Sciences, Bandar Abbas, Iran

\section{Mohadeseh Khaladeh}

Student Research Committee, Faculty of Medicine, Homozgan University of Medical Sciences, Bandar Abbas, Iran

\section{Hadi Yousefi}

Department of Epidemiology and Biostatistics, Faculty of Medicine, Hormozgan University of Medical Sciences, Bandar Abbas, Iran

Masoumeh Kheirandish ( $\sim$ kheirandishm@yahoo.com )

Endocrinology and Metabolism Research Center, Hormozgan University of Medical Sciences, Bandar Abbas, Iran https://orcid.org/0000-0002-7355-4456

\section{Research}

Keywords: triglyceride, high-density lipoprotein, low-density lipoprotein, cholesterol, Prospective Epidemiological Research Studies in IrAN (PERSIAN)

Posted Date: August 12th, 2020

DOI: https://doi.org/10.21203/rs.3.rs-56630/v1

License: (c) (1) This work is licensed under a Creative Commons Attribution 4.0 International License. Read Full License 


\section{Abstract}

Background: Dyslipidemia, a major risk factor for cardiovascular diseases, has become a global issue. Due to geographic and demographic variations in the prevalence of dyslipidemia, this study aimed to evaluate dyslipidemia and its associated factors in women of the Bandare-Kong Cohort Study (BKNCD).

Methods: This study was conducted on women from the population-based BKNCD, as part of the Prospective Epidemiological Research Studies in IrAN (PERSIAN). Sociodemographic data, medical history, and anthropometric indices were collected. Diastolic blood pressure, systolic blood pressure, fasting plasma glucose, serum triglyceride (TG), total cholesterol (TC), high-density lipoprotein (HDL), and low-density lipoprotein (LDL) were measured. Daily calorie intake and weekly metabolic equivalent of tasks were also recorded. Dyslipidemia was defined as any lipid abnormality based on the Adult Treatment Panel III criteria.

Results: From the 2223 women in this study (mean age: $48.28 \pm 9.26$ years), dyslipidemia was observed in 1884 (84.8\%). High TC was the most common lipid abnormality (53.9\%) followed by low HDL (50.8\%). Dyslipidemia was most prevalent among women aged 65-70 years, the widowed, illiterate, unemployed, and overweight, with very low socioeconomic status, diabetes, and high waist circumference, hypertension, those using hookah and living in urban areas. Logistic regression revealed that only women with high WHR were at increased risk of dyslipidemia (OR=2.48, 95\% Cl:1.75-3.53, $\mathrm{P}<0.001)$. Having a high school diploma (OR=0.57, 95\% Cl:0.37-0.89, $\mathrm{P}=0.015)$ and living in rural areas (OR=0.68 95\% Cl:0.49$0.96, \mathrm{P}=0.028$ ) were protective.

Conclusions: Dyslipidemia was highly prevalent in women of BKNCD. High WHR appears to be the only significant risk factor for dyslipidemia.

\section{Introduction}

Dyslipidemia, a disorder of lipid metabolism, is clinically defined as the presence of one of the following abnormalities: elevated plasma triglycerides (TG), elevated total cholesterol (TC), high levels of lowdensity lipoprotein (LDL), and decreased high-density lipoprotein (HDL) (1). With the increasing prevalence of dyslipidemia, mostly due to adverse changes in lifestyle including dietary changes, the more sedentary lifestyle, and reduced physical activity, it has become a global public health issue $(2,3)$. There is substantial evidence that dyslipidemia is associated with an increased risk of cardiovascular disease (CVD) (4). According to the World Health Organization (WHO) estimates, dyslipidemia, especially high TC, is responsible for 2.6 million deaths annually and 29.7 million disability-adjusted life years (DALYS) worldwide (5).

The prevalence of dyslipidemia varies in different regions, with hypercholesterolemia ranging from 22.6$54 \%$ across Africa, South East Asia, Europe, and America (5). Studies in Iran have also reported the prevalence of dyslipidemia: the prevalence of hypertriglyceridemia, hypercholesterolemia, high LDL, and low HDL ranged $14-40.6 \%, 14-61 \%, 13.4-45.5 \%$, and 5-73\%, respectively (6-9). In addition, it has been 
demonstrated in many parts of the world including Iran, that dyslipidemia can be influenced by numerous factors including socio-economic status, level of fat intake, obesity, and gender $(7,10-13)$. Dyslipidemia is a modifiable risk factor for the development of type 2 diabetes, atherosclerosis, CVD, and stroke; early effective management of patients with dyslipidemia can decrease the incidence and burden of the abovementioned conditions (14-19).

Metabolism in women can be affected by alterations in hormonal levels throughout their lives, in either the premenopausal or postmenopausal period (20). It has been reported that serum TC levels in women increases with age; however, this occurs more gradually compared to men (12). Yet, it increases at a higher rate after the age of 44 years, probably as a result of loss of estrogen in the postmenopausal period and decreased activity of LDL receptors (21).

With regard to regional differences in the prevalence of dyslipidemia and gender variations in this respect, and taken into consideration the modifiable nature of dyslipidemia for prevention and control of the disease burden, as well as specific hormonal effects in females, it would be extremely important to be aware of the prevalence and potential influencing factors of this condition in women. Thus, we aimed to evaluate dyslipidemia and its associated factors in women of the PERSIAN Bandare-Kong Cohort Study.

\section{Methods}

\section{Participants}

We evaluated the women of the PERSIAN Bandare Kong Cohort Study, a prospective, population-based cohort study in Bandare-Kong, Iran, which has been previously described in detail (22). This cohort study includes 2334 women aged 35-70 years, recruited between November 17, 2016 and November 22, 2018, from Hormozgan province, southern Iran, as part of the Prospective Epidemiological Research Studies in IrAN (PERSIAN). Written informed consent was obtained from all the participants. After exclusion of pregnant women, those taking lipid-lowering medications, and incomplete records, 2223 women were included in the final analysis.

\section{Study design}

The BKNCD cohort study is part of the PERSIAN (Prospective Epidemiological Research Studies in IrAN) Cohort. Sociodemographic data were collected using a face-to-face interview by trained interviewers. Age, education, marital status, place of residence, and hookah use were recorded. Data regarding occupation, type of residence ownership, home size/area, trips, and other possessions including cars, computers, dishwasher, etc. were used to determine socioeconomic status (SES) by means of principal component analysis. Daily calorie intake was calculated using daily ingested foods reported by the participants and their calorie content. Daily and weekly energy expenditure were determined using the metabolic equivalent of tasks (METs). 
Weight was measured with a digital scale (measurement accuracy of $0.5 \mathrm{~kg}$ ), with subjects in minimum clothing and without shoes. Height was measured with subjects standing shoeless and with their shoulders set normally. Waist circumference (WC) was measured twice for each participant and the average was recorded. WC was measured at the end of several consecutive natural breaths, at a level parallel to the floor, the midpoint between the top of the iliac crest and the inferior margin of the last palpable rib in the midaxillary line. Hip circumference $(\mathrm{HC})$ was measured at the largest circumference of the buttocks, at a parallel level to the floor. All measurements were done with the same stretch-resistant tape to the nearest $0.5 \mathrm{~cm}$. Subjects were standing upright during the measurements, with arms relaxed at the side, feet evenly spread apart and body weight evenly distributed (23). Waist-to-hip ratio (WHR) was calculated as WC divided by $\mathrm{HC}$ to the nearest 0.01 . WHO cut-off for substantially increased risk of metabolic complications in women are: WC $>88 \mathrm{~cm}$ and WHR $\geq 0.85$ (23). According to the study by Azizi et al. the cut-off value of WC for the Iranian population is $W C \geq 95 \mathrm{~cm}$ for both men and women (24). No WHR cut-off has been established for the Iranian population; therefore, the WHO cut-off for WHR was used.

BMI was calculated as weight in kilograms divided by the square of the person's height in meters to the nearest 0.01 . According to the $\mathrm{WHO}$ guidelines, $\mathrm{BMI}<18.5 \mathrm{~kg} / \mathrm{m} 2$ was considered as underweight, $18.5 \leq$ $\mathrm{BMI}<25$ as normal, $25 \leq \mathrm{BMI}<30$ as overweight, and $\mathrm{BMI} \geq 30$ as obese (25).

Blood pressure (BP) was measured using a standard mercury sphygmomanometer after 5 minutes of rest with an appropriate cuff size for the upper-arm circumference, in the seated position, with feet on the floor, and arm supported at heart level. The average of two measurements made at least 5 min apart was used for analysis. Hypertension was defined as sustained blood pressure $\geq 140 / 90 \mathrm{mmHg}(26)$ or treatment with anti-hypertensive medications. Elevated values $(\geq 140 / 90 \mathrm{mmHg})$ were confirmed on a separate day.

Venous blood samples were collected following overnight 8-hour fasting and fasting plasma glucose (FPG) was measured. Plasma glucose measurements were done using the glucose oxidase method. According to the American Diabetes Association (ADA) criteria, diabetes was defined as an FPG $\geq$ $126 \mathrm{mg} / \mathrm{dl}$, confirmed in a repeat test, or treatment with glucose-lowering agents (27). Venous blood samples were collected on another day following overnight 12-hour fasting and TC, TG, LDL, and HDL were measured for each participant using the enzymatic method. $L D L<100 \mathrm{mg} / \mathrm{dl}$ was considered optimal, $100-129 \mathrm{mg} / \mathrm{dl}$ near or above optimal, 130-159 borderline high, 160-189 high, and $\geq 190$ very high. TC $<200 \mathrm{mg} / \mathrm{dl}$ was considered desirable, 200-239 borderline high, and $\geq 240$ high. Low HDL was defined as $\mathrm{HDL}<50 \mathrm{mg} / \mathrm{dl}$ in women and $\mathrm{HDL} \geq 60 \mathrm{mg} / \mathrm{dl}$ was considered high. TG $<150 \mathrm{mg} / \mathrm{dl}$ was considered normal, 150-199 borderline high, 200-499 high, and $\geq 500$ very high. Based on the Adult Treatment Panel (ATP III) criteria (28), dyslipidemia was defined as the presence of one or more of the following disorders:

1. $T C \geq 200 \mathrm{mg} / \mathrm{dl}$

2. $T G \geq 150 \mathrm{mg} / \mathrm{dl}$ 
3. $\mathrm{LDL} \geq 130 \mathrm{mg} / \mathrm{dl}$

4. $\mathrm{HDL}<50 \mathrm{mg} / \mathrm{dl}$ (in women)

\section{Data analysis}

Statistical Package for the Social Sciences (SPSS) software (version 25.0, Armonk, NY: IBM Corp.) was used for data analysis. Mean, standard deviation, frequency, and percentages were used to describe the results. Logistic regression model was used to examine the correlation of dyslipidemia and its components with associated factors. Linear regression was used to determine the predictive power of associated factors on lipid profile components. P-values of equal to or less than 0.05 were regarded as statistically significant.

\section{Results}

From the 2223 women evaluated in this study (with mean age of $48.28 \pm 9.26$ ), dyslipidemia was observed in 1884 (84.8\%). Mean values of TC, TG, HDL, and LDL were $204.72 \pm 42.39 \mathrm{mg} / \mathrm{dl}, 127.19 \pm$ $68.73 \mathrm{mg} / \mathrm{dl}, 50.37 \pm 10.83 \mathrm{mg} / \mathrm{dl}$, and $129.16 \pm 35.52 \mathrm{mg} / \mathrm{dl}$, respectively. High TC was the most common lipid abnormality (53.9\%) followed by low HDL (50.8\%), high LDL (48\%), and high TG (26.2\%). One, two, three, and four abnormal lipid components were observed in $26.6 \%, 30.2 \%, 19.9 \%$, and $8.1 \%$, respectively. High TG (individually, with other components being normal), high TC, low HDL, and high LDL were exclusively seen in $1.2 \%, 4.3 \%, 20.8 \%$, and $0.3 \%$, respectively. In general, most participants were aged $35-44$ years $(22.3 \%)$. They were mostly married $(83.1 \%)$, and illiterate $(55.1 \%)$. Most of them lived in urban areas $(84.3 \%)$, had very low socioeconomic status $(23.3 \%)$, and were unemployed $(84.8 \%)$. Moreover, $13.5 \%$ used hookah. With regard to medical history and anthropometric indices, $20.9 \%$ had diabetes, $30 \%$ had hypertension, most participants were overweight (39\%), $87.4 \%$ had high WHR, $76.4 \%$ had high WC based on WHO cut-off, and $53.6 \%$ based on Iranian-specific cut-off.

Prevalence of dyslipidemia and individual lipid abnormalities are demonstrated in Table 1. The prevalence of dyslipidemia was the highest in women aged 65-70 years, while high TC and high LDL were most prevalent in 60-64 age group. On the other hand, high TG and low HDL were most prevalent in 50-54, and 40-44 age groups, respectively. Except for low HDL which was more prevalent among married women, dyslipidemia and all other lipid abnormalities were highest in widowed participants. Aside from low HDL, which was the highest in those with an associate's degree, illiterate participants had the highest rate of dyslipidemia and lipid abnormalities. Apart from high TG, most prevalent in those living in rural areas, dyslipidemia and other lipid abnormalities were most prevalent in those living in urban areas. Details of lipid abnormalities are shown in Table 2.

Logistic regression revealed that high WHR was consistently correlated with dyslipidemia and all of its components. Women living in rural areas were at increased risk of dyslipidemia compared to those living in urban areas, while having a high school diploma was protective against dyslipidemia. Age of 50-54 years, overweight, obesity, diabetes, and were associated with high TG. Women aged 45-64 years were at 
increased risk of having high TC; however, surprisingly diabetes and hookah were protective in this regard. On the contrary, hookah, as well as diabetes and 50-64 years of age were risk factors for low $\mathrm{HDL}$, while high or very high socioeconomic status and living in rural areas were protective. Women aged 45-64 years were also at increased risk of having high LDL. High WC based on WHO cutoff was another risk factor for high LDL. Nevertheless, living in rural areas and diabetes were negatively correlated with high LDL (Table 3).

Linear regression ("enter" method) showed that every $1 \mathrm{~kg} / \mathrm{m}^{2}$ increase in BMI led to $2.51,1.56$, and $0.94 \mathrm{mg} / \mathrm{dl}$ increase in TG, TC, and LDL, respectively. Every $1 \mathrm{mg} / \mathrm{dl}$ increase in FPG and every $1 \mathrm{mmHg}$ increase in systolic blood pressure (SBP) led to 0.22 and $0.41 \mathrm{mg} / \mathrm{dl}$ increase in TG, respectively. Every 1 year advance in age and $1 \mathrm{mmHg}$ elevation in diastolic blood pressure (DBP) led to 0.59 and $0.31 \mathrm{mg} / \mathrm{dl}$ increase in TC, respectively. Weight, HC, and WHR were negatively correlated with HDL. Age was also positively associated with LDL. Besides, altogether age, years of education, weight, WC, HC, WHR, BMI, FPG, SBP, DBP, daily calorie intake, and weekly METs could predict $14 \%, 6 \%, 7 \%$, and $3 \%$ of the variance of TG, TC, HDL, and LDL, respectively (Table 4). 
Table 1

Prevalence of dyslipidemia and individual lipid abnormalities

\begin{tabular}{|c|c|c|c|c|c|c|c|}
\hline \multicolumn{2}{|l|}{ Variable } & \multirow{2}{*}{$\begin{array}{l}\text { Total } \\
\mathrm{N}(\%)\end{array}$} & \multirow{2}{*}{$\begin{array}{l}\text { Dyslipidemia } \\
\%\end{array}$} & \multirow{2}{*}{$\begin{array}{l}\text { High } \\
\text { TG } \\
\%\end{array}$} & \multirow{2}{*}{$\begin{array}{l}\text { High } \\
\text { TC } \\
\%\end{array}$} & \multirow{2}{*}{$\begin{array}{l}\text { Low } \\
\text { HDL } \\
\%\end{array}$} & \multirow{2}{*}{$\begin{array}{l}\text { High } \\
\text { LDL }\end{array}$} \\
\hline & & & & & & & \\
\hline \multirow[t]{7}{*}{$\begin{array}{l}\text { Age groups } \\
\text { (years) }\end{array}$} & $35-39$ & $\begin{array}{l}496 \\
(22.3)\end{array}$ & 79.6 & 16.9 & 41.5 & 53.0 & 38.5 \\
\hline & $40-44$ & $\begin{array}{l}410 \\
(18.4)\end{array}$ & 81.5 & 23.2 & 44.9 & 56.8 & 40.2 \\
\hline & $45-49$ & $\begin{array}{l}384 \\
(17.3)\end{array}$ & 87.5 & 26.3 & 58.1 & 53.9 & 49.7 \\
\hline & $50-54$ & $\begin{array}{l}327 \\
(14.7)\end{array}$ & 89.0 & 34.6 & 61.8 & 47.4 & 55.4 \\
\hline & $55-59$ & $\begin{array}{l}293 \\
(13.2)\end{array}$ & 85.3 & 32.8 & 63.5 & 42.3 & 54.6 \\
\hline & $60-64$ & $179(8.1)$ & 87.2 & 27.9 & 67.6 & 44.1 & 63.1 \\
\hline & $65-70$ & $134(6.0)$ & 91.0 & 32.1 & 56.7 & 50.7 & 50.0 \\
\hline \multirow[t]{4}{*}{ Marital status } & Single & $72(3.2)$ & 76.4 & 22.2 & 47.2 & 41.7 & 38.9 \\
\hline & Married & $\begin{array}{l}1848 \\
(83.1)\end{array}$ & 84.8 & 25.6 & 52.9 & 51.4 & 47.3 \\
\hline & Widowed & $\begin{array}{l}241 \\
(10.8)\end{array}$ & 87.6 & 32.4 & 62.2 & 50.2 & 57.3 \\
\hline & Divorced & $62(2.8)$ & 80.6 & 22.6 & 58.1 & 46.8 & 43.5 \\
\hline \multirow[t]{6}{*}{ Education } & Illiterate & $\begin{array}{l}1225 \\
(55.1)\end{array}$ & 87.6 & 28.8 & 59.3 & 48.7 & 52.3 \\
\hline & $\begin{array}{l}\text { Elementary } \\
\text { school }\end{array}$ & $\begin{array}{l}506 \\
(22.8)\end{array}$ & 85.2 & 25.7 & 49.2 & 57.3 & 45.7 \\
\hline & Middle school & 175 (7.9) & 79.4 & 23.4 & 48.6 & 53.1 & 41.7 \\
\hline & $\begin{array}{l}\text { High school } \\
\text { diploma }\end{array}$ & $199(9.0)$ & 75.9 & 20.1 & 44.7 & 46.2 & 37.2 \\
\hline & $\begin{array}{l}\text { Associate's } \\
\text { degree }\end{array}$ & $29(1.3)$ & 82.8 & 17.2 & 44.8 & 58.6 & 44.8 \\
\hline & $\begin{array}{l}\text { Bachelor's } \\
\text { degree }\end{array}$ & $74(3.3)$ & 77.0 & 14.9 & 40.5 & 47.3 & 41.9 \\
\hline
\end{tabular}

Abbreviations: $\mathrm{N}$, number; TG, triglyceride; TC, total cholesterol; HDL, high-density lipoprotein; LDL, low-density lipoprotein; SES, socioeconomic status; BMI, body mass index; WHO, World Health Organization; WC, waist circumference; WHR, waist-to-hip ratio. 


\begin{tabular}{|c|c|c|c|c|c|c|c|}
\hline \multirow[t]{3}{*}{ Variable } & & Total & Dyslipidemia & $\begin{array}{l}\text { High } \\
\text { TG }\end{array}$ & $\begin{array}{l}\text { High } \\
\text { TC }\end{array}$ & $\begin{array}{l}\text { Low } \\
\text { HDL }\end{array}$ & $\begin{array}{l}\text { High } \\
\text { LDL }\end{array}$ \\
\hline & Master's degree & $14(0.6)$ & 64.3 & 14.3 & 42.9 & 42.9 & 35.7 \\
\hline & $\mathrm{PhD}$ & $1(0.0)$ & 0.0 & 0.0 & 0.0 & 0.0 & 0.0 \\
\hline \multirow[t]{2}{*}{$\begin{array}{l}\text { Place of } \\
\text { residence }\end{array}$} & Urban & $\begin{array}{l}1874 \\
(84.3)\end{array}$ & 85.0 & 25.9 & 54.1 & 52.9 & 48.6 \\
\hline & Rural & $\begin{array}{l}349 \\
(15.7)\end{array}$ & 83.4 & 27.5 & 53.0 & 39.5 & 45.0 \\
\hline \multirow[t]{5}{*}{ SES } & Very low & $\begin{array}{l}517 \\
(23.3)\end{array}$ & 85.7 & 27.9 & 51.6 & 55.7 & 46.6 \\
\hline & Low & $\begin{array}{l}461 \\
(20.7)\end{array}$ & 84.2 & 26.9 & 56.2 & 50.3 & 50.8 \\
\hline & Average & $\begin{array}{l}445 \\
(20.0)\end{array}$ & 85.4 & 24.7 & 54.8 & 51.9 & 49.0 \\
\hline & High & $\begin{array}{l}444 \\
(20.0)\end{array}$ & 84.7 & 25.9 & 53.2 & 48.6 & 47.1 \\
\hline & Very high & $\begin{array}{l}356 \\
(16.0)\end{array}$ & 83.4 & 25.0 & 53.9 & 45.5 & 46.6 \\
\hline \multirow[t]{2}{*}{ Occupation } & Unemployed & $\begin{array}{l}1884 \\
(84.8)\end{array}$ & 85.6 & 27.0 & 55.3 & 50.7 & 49.2 \\
\hline & Employed & $\begin{array}{l}339 \\
(15.2)\end{array}$ & 80.2 & 21.5 & 46.3 & 51.0 & 41.9 \\
\hline \multirow[t]{2}{*}{ Hookah } & No & $\begin{array}{l}1922 \\
(86.5)\end{array}$ & 84.6 & 26.2 & 55.0 & 49.5 & 48.5 \\
\hline & Yes & $\begin{array}{l}301 \\
(13.5)\end{array}$ & 85.7 & 25.9 & 46.8 & 58.8 & 44.9 \\
\hline \multirow[t]{2}{*}{ Diabetes } & No & $\begin{array}{l}1759 \\
(79.1)\end{array}$ & 83.6 & 21.6 & 53.3 & 49.9 & 48.0 \\
\hline & Yes & $\begin{array}{l}464 \\
(20.9)\end{array}$ & 89.2 & 43.5 & 56.0 & 54.1 & 48.1 \\
\hline \multirow[t]{2}{*}{ Hypertension } & No & $\begin{array}{l}1556 \\
(70.0)\end{array}$ & 83.5 & 21.8 & 50.6 & 51.9 & 45.8 \\
\hline & Yes & $\begin{array}{l}667 \\
(30.0)\end{array}$ & 87.7 & 36.4 & 61.6 & 48.3 & 53.4 \\
\hline BMI & Underweight & $47(2.1)$ & 63.8 & 2.1 & 42.6 & 31.9 & 34.0 \\
\hline
\end{tabular}

Abbreviations: $\mathrm{N}$, number; TG, triglyceride; TC, total cholesterol; HDL, high-density lipoprotein; LDL, low-density lipoprotein; SES, socioeconomic status; BMI, body mass index; WHO, World Health Organization; WC, waist circumference; WHR, waist-to-hip ratio. 


\begin{tabular}{|c|c|c|c|c|c|c|c|}
\hline \multicolumn{2}{|l|}{ Variable } & \multirow{2}{*}{$\begin{array}{l}\text { Total } \\
630 \\
(28.3)\end{array}$} & \multirow{2}{*}{$\begin{array}{l}\text { Dyslipidemia } \\
80.2\end{array}$} & \multirow{2}{*}{$\begin{array}{l}\text { High } \\
\text { TG } \\
16.8\end{array}$} & \multirow{2}{*}{$\begin{array}{l}\text { High } \\
\text { TC } \\
50.2\end{array}$} & \multirow{2}{*}{$\begin{array}{l}\text { Low } \\
\text { HDL } \\
45.1\end{array}$} & \multirow{2}{*}{$\begin{array}{l}\begin{array}{l}\text { High } \\
\text { LDL }\end{array} \\
44.8\end{array}$} \\
\hline & Normal & & & & & & \\
\hline & Overweight & $\begin{array}{l}867 \\
(39.0)\end{array}$ & 87.2 & 29.5 & 56.2 & 54.2 & 50.6 \\
\hline & Obese & $\begin{array}{l}679 \\
(30.5)\end{array}$ & 87.3 & 32.3 & 55.2 & 53.0 & 48.7 \\
\hline \multirow[t]{2}{*}{ WHO WC } & Normal & $\begin{array}{l}525 \\
(23.6)\end{array}$ & 75.8 & 13.3 & 46.5 & 42.9 & 38.7 \\
\hline & High & $\begin{array}{l}1698 \\
(76.4)\end{array}$ & 87.5 & 30.2 & 56.2 & 53.2 & 50.9 \\
\hline \multirow[t]{2}{*}{ Iranian WC } & Normal & $\begin{array}{l}1031 \\
(46.4)\end{array}$ & 80.9 & 18.0 & 51.6 & 46.8 & 46.0 \\
\hline & High & $\begin{array}{l}1192 \\
(53.6)\end{array}$ & 88.1 & 33.2 & 55.9 & 54.3 & 49.8 \\
\hline \multirow[t]{2}{*}{ WHO WHR } & Normal & $\begin{array}{l}279 \\
(12.6)\end{array}$ & 67.4 & 7.2 & 39.8 & 38.4 & 32.6 \\
\hline & High & $\begin{array}{l}1944 \\
(87.4)\end{array}$ & 87.2 & 28.9 & 55.9 & 52.6 & 50.3 \\
\hline $\begin{array}{l}\text { Abbreviatio } \\
\text { low-density } \\
\text { Organizatio }\end{array}$ & $\begin{array}{l}\text { umber; TG } \\
\text { tein; SES, } \\
\text { waist circu }\end{array}$ & $\begin{array}{l}\text { de; TC } \\
\text { omic s } \\
\text { WHR, }\end{array}$ & $\begin{array}{l}\text { cholesterol; } \\
\text { BMl, body m } \\
\text { to-hip ratio. }\end{array}$ & $\begin{array}{l}\mathrm{L} \text {, hig } \\
\text { ss ind }\end{array}$ & $\begin{array}{l}\text { nsity } \\
\text { NHO, }\end{array}$ & $\begin{array}{l}\text { oprot } \\
\text { rld H }\end{array}$ & $\mathrm{DL}$ \\
\hline
\end{tabular}


Table 2

Details of lipid abnormalities based on ATP III cut-offs

\begin{tabular}{|c|c|c|c|c|}
\hline Variable & Range & & $\mathbf{N}$ & $\%$ \\
\hline \multirow[t]{4}{*}{ TG } & $<150 \mathrm{mg} / \mathrm{dl}$ & Normal & 1641 & 73.8 \\
\hline & $150-199$ mg/dl & Borderline high & 336 & 15.1 \\
\hline & $200-499 \mathrm{mg} / \mathrm{dl}$ & High & 240 & 10.8 \\
\hline & $\geq 500 \mathrm{mg} / \mathrm{dl}$ & Very high & 6 & 0.3 \\
\hline \multirow[t]{3}{*}{ TC } & $<200 \mathrm{mg} / \mathrm{dl}$ & Desirable & 1025 & 46.1 \\
\hline & $200-239 \mathrm{mg} / \mathrm{dl}$ & Borderline high & 787 & 35.4 \\
\hline & $\geq 240 \mathrm{mg} / \mathrm{dl}$ & High & 411 & 18.5 \\
\hline \multirow[t]{3}{*}{ HDL } & $<50 \mathrm{mg} / \mathrm{dl}$ & Low & 1129 & 50.8 \\
\hline & $50-59 \mathrm{mg} / \mathrm{dl}$ & Normal & 653 & 29.4 \\
\hline & $\geq 60 \mathrm{mg} / \mathrm{dl}$ & High & 441 & 19.8 \\
\hline \multirow[t]{5}{*}{ LDL } & $<100 \mathrm{mg} / \mathrm{dl}$ & Optimal & 467 & 21.0 \\
\hline & $100-129 \mathrm{mg} / \mathrm{dl}$ & Near or above optimal & 688 & 30.9 \\
\hline & $130-159 \mathrm{mg} / \mathrm{dl}$ & Borderline high & 674 & 30.3 \\
\hline & $160-189$ mg/dl & High & 272 & 12.2 \\
\hline & $\geq 190 \mathrm{mg} / \mathrm{dl}$ & Very high & 122 & 5.5 \\
\hline
\end{tabular}


Table 3

Logistic regression analysis of dyslipidemia and individual lipid abnormalities

Variable

\begin{tabular}{|c|c|c|c|c|c|c|}
\hline & & OR $(95 \% \mathrm{Cl})$ & $\begin{array}{l}\text { OR }(95 \% \\
\mathrm{Cl})\end{array}$ & $\begin{array}{l}\text { OR }(95 \% \\
\mathrm{Cl})\end{array}$ & $\begin{array}{l}\mathrm{OR}(95 \% \\
\mathrm{Cl})\end{array}$ & $\begin{array}{l}\text { OR }(95 \% \\
\mathrm{Cl})\end{array}$ \\
\hline \multirow{7}{*}{$\begin{array}{l}\text { Age groups } \\
\text { (years) }\end{array}$} & $35-39 *$ & 1.00 & 1.00 & 1.00 & 1.00 & 1.00 \\
\hline & $40-44$ & $\begin{array}{l}0.82(0.58- \\
1.18)\end{array}$ & $\begin{array}{l}1.26 \\
(0.89- \\
1.78)\end{array}$ & $\begin{array}{l}1.04 \\
(0.79- \\
1.37)\end{array}$ & $\begin{array}{l}1.00 \\
(0.76- \\
1.33)\end{array}$ & $\begin{array}{l}0.96 \\
(0.73- \\
1.28)\end{array}$ \\
\hline & $45-49$ & $\begin{array}{l}1.11(0.73- \\
1.70)\end{array}$ & $\begin{array}{l}1.22 \\
(0.85- \\
1.76)\end{array}$ & $\begin{array}{l}1.65 \\
(1.22- \\
2.23)^{\star \star}\end{array}$ & $\begin{array}{l}0.86 \\
(0.63- \\
1.16)\end{array}$ & $\begin{array}{l}1.35 \\
(1.00- \\
1.82)^{\star \star}\end{array}$ \\
\hline & $50-54$ & $\begin{array}{l}1.25(0.78- \\
1.98)\end{array}$ & $\begin{array}{l}1.71 \\
(1.18- \\
2.49)^{\star \star}\end{array}$ & $\begin{array}{l}1.91 \\
(1.39- \\
2.64)^{\star \star}\end{array}$ & $\begin{array}{l}0.63 \\
(0.46- \\
0.87)^{\star \star}\end{array}$ & $\begin{array}{l}1.70 \\
(1.23- \\
2.34)^{\star \star}\end{array}$ \\
\hline & $55-59$ & $\begin{array}{l}0.82(0.51- \\
1.33)\end{array}$ & $\begin{array}{l}1.35 \\
(0.90- \\
2.02)\end{array}$ & $\begin{array}{l}2.03 \\
(1.43- \\
2.87)^{\star \star}\end{array}$ & $\begin{array}{l}0.50 \\
(0.35- \\
0.71)^{\star \star}\end{array}$ & $\begin{array}{l}1.64 \\
(1.16- \\
2.32)^{\star \star}\end{array}$ \\
\hline & $60-64$ & $\begin{array}{l}0.92(0.51- \\
1.64)\end{array}$ & $\begin{array}{l}1.05 \\
(0.65- \\
1.70)\end{array}$ & $\begin{array}{l}2.39 \\
(1.57- \\
3.63)^{\star \star}\end{array}$ & $\begin{array}{l}0.52 \\
(0.35- \\
0.79)^{\star \star}\end{array}$ & $\begin{array}{l}2.24 \\
(1.48- \\
3.39)^{\star \star}\end{array}$ \\
\hline & $65-70$ & $\begin{array}{l}1.42(0.68- \\
2.92)\end{array}$ & $\begin{array}{l}1.20 \\
(0.71- \\
2.03)\end{array}$ & $\begin{array}{l}1.43 \\
(0.91- \\
2.27)\end{array}$ & $\begin{array}{l}0.71 \\
(0.45- \\
1.13)\end{array}$ & $\begin{array}{l}1.26 \\
(0.80- \\
1.99)\end{array}$ \\
\hline \multirow{4}{*}{$\begin{array}{l}\text { Marital } \\
\text { status }\end{array}$} & Single* & 1.00 & 1.00 & 1.00 & 1.00 & 1.00 \\
\hline & Married & $\begin{array}{l}1.13(0.61- \\
2.07)\end{array}$ & $\begin{array}{l}0.76 \\
(0.41- \\
1.40)\end{array}$ & $\begin{array}{l}0.86 \\
(0.52- \\
1.42)\end{array}$ & $\begin{array}{l}1.38 \\
(0.83- \\
2.28)\end{array}$ & $\begin{array}{l}1.04 \\
(0.62- \\
1.73)\end{array}$ \\
\hline & Widowed & $\begin{array}{l}1.17(0.56- \\
2.45)\end{array}$ & $\begin{array}{l}0.96 \\
(0.48- \\
1.92)\end{array}$ & $\begin{array}{l}1.12 \\
(0.63- \\
1.99)\end{array}$ & $\begin{array}{l}1.33 \\
(0.74- \\
2.36)\end{array}$ & $\begin{array}{l}1.36 \\
(0.76- \\
2.44)\end{array}$ \\
\hline & Divorced & $\begin{array}{l}0.82(0.34- \\
2.00)\end{array}$ & $\begin{array}{l}0.71 \\
(0.29- \\
1.70)\end{array}$ & $\begin{array}{l}1.17 \\
(0.57- \\
2.39)\end{array}$ & $\begin{array}{l}0.99 \\
(0.48- \\
2.04)\end{array}$ & $\begin{array}{l}0.90 \\
(0.44- \\
1.86)\end{array}$ \\
\hline Education & Illiterate* & 1.00 & 1.00 & 1.00 & 1.00 & 1.00 \\
\hline
\end{tabular}

Abbreviations: OR, odds ratio; $\mathrm{Cl}$, confidence interval; TG, triglyceride; TC, total cholesterol; $\mathrm{HDL}$, highdensity lipoprotein; LDL, low-density lipoprotein; SES, socioeconomic status; BMI, body mass index; WHO, World Health Organization; WC, waist circumference; WHR, waist-to-hip ratio.

*Reference categories.

**Statistically significant (P-value $<0.05)$.

High TG High TC Low HDL High LDL

$\begin{array}{lllll}\text { Dyslipidemia } & \text { High TG } & \text { High TC } & \text { Low HDL } & \text { High LDL } \\ & & & & \\ \text { OR (95\% Cl) } & \text { OR (95\% } & \text { OR (95\% } & \text { OR (95\% } & \text { OR (95\% } \\ & \text { Cl) } & \text { Cl) } & \text { Cl) } & \text { Cl) }\end{array}$

Age grou
(years) 


\begin{tabular}{|c|c|c|c|c|c|c|}
\hline \multicolumn{2}{|l|}{ Variable } & \multirow{2}{*}{$\begin{array}{l}\text { Dyslipidemia } \\
0.88(0.63- \\
1.25)\end{array}$} & \multirow{2}{*}{$\begin{array}{l}\text { High TG } \\
\begin{array}{l}1.10 \\
(0.84- \\
1.44)\end{array}\end{array}$} & \multirow{2}{*}{$\begin{array}{l}\text { High TC } \\
0.86 \\
(0.67- \\
1.08)\end{array}$} & \multirow{2}{*}{$\begin{array}{l}\text { Low HDL } \\
1.20 \\
(0.95- \\
1.53)\end{array}$} & \multirow{2}{*}{$\begin{array}{l}\text { High LDL } \\
0.95 \\
(0.75- \\
1.20)\end{array}$} \\
\hline & $\begin{array}{l}\text { Elementary } \\
\text { school }\end{array}$ & & & & & \\
\hline & $\begin{array}{l}\text { Middle } \\
\text { school }\end{array}$ & $\begin{array}{l}0.67(0.42- \\
1.06)\end{array}$ & $\begin{array}{l}1.20 \\
(0.79- \\
1.83)\end{array}$ & $\begin{array}{l}0.98 \\
(0.69- \\
1.40)\end{array}$ & $\begin{array}{l}1.00 \\
(0.70- \\
1.43)\end{array}$ & $\begin{array}{l}0.90 \\
(0.63- \\
1.28)\end{array}$ \\
\hline & $\begin{array}{l}\text { High school } \\
\text { diploma }\end{array}$ & $\begin{array}{l}0.57(0.37- \\
0.89)^{\star \star}\end{array}$ & $\begin{array}{l}1.11 \\
(0.72- \\
1.70)\end{array}$ & $\begin{array}{l}0.83 \\
(0.59- \\
1.18)\end{array}$ & $\begin{array}{l}0.82 \\
(0.58- \\
1.16)\end{array}$ & $\begin{array}{l}0.77 \\
(0.54- \\
1.10)\end{array}$ \\
\hline & $\begin{array}{l}\text { Associate's } \\
\text { degree }\end{array}$ & $\begin{array}{l}1.00(0.35- \\
2.85)\end{array}$ & $\begin{array}{l}1.02 \\
(0.36- \\
2.88)\end{array}$ & $\begin{array}{l}0.87 \\
(0.39- \\
1.90)\end{array}$ & $\begin{array}{l}1.53 \\
(0.69- \\
3.40)\end{array}$ & $\begin{array}{l}1.13 \\
(0.51- \\
2.49)\end{array}$ \\
\hline & $\begin{array}{l}\text { Bachelor's } \\
\text { degree }\end{array}$ & $\begin{array}{l}0.69(0.35- \\
1.37)\end{array}$ & $\begin{array}{l}0.87 \\
(0.42- \\
1.82)\end{array}$ & $\begin{array}{l}0.76 \\
(0.44- \\
1.32)\end{array}$ & $\begin{array}{l}0.98 \\
(0.57- \\
1.69)\end{array}$ & $\begin{array}{l}1.09 \\
(0.63- \\
1.89)\end{array}$ \\
\hline & $\begin{array}{l}\text { Master's } \\
\text { degree }\end{array}$ & $\begin{array}{l}0.34(0.10- \\
1.14)\end{array}$ & $\begin{array}{l}0.70 \\
(0.13- \\
3.53)\end{array}$ & $\begin{array}{l}0.80 \\
(0.26- \\
2.47)\end{array}$ & $\begin{array}{l}0.83 \\
(0.27- \\
2.55)\end{array}$ & $\begin{array}{l}0.81 \\
(0.25- \\
2.56)\end{array}$ \\
\hline & $\mathrm{PhD}$ & 0.00 & 0.00 & 0.00 & 0.00 & 0.00 \\
\hline \multirow{2}{*}{$\begin{array}{l}\text { Place of } \\
\text { residence }\end{array}$} & Urban* & 1.00 & 1.00 & 1.00 & 1.00 & 1.00 \\
\hline & Rural & $\begin{array}{l}0.68(0.49- \\
0.96)^{\star \star}\end{array}$ & $\begin{array}{l}0.94 \\
(0.71- \\
1.24)\end{array}$ & $\begin{array}{l}0.84 \\
(0.66- \\
1.08)\end{array}$ & $\begin{array}{l}0.54 \\
(0.42- \\
0.69)^{\star \star}\end{array}$ & $\begin{array}{l}0.76 \\
(0.59- \\
0.97)^{\star \star}\end{array}$ \\
\hline \multirow[t]{4}{*}{ SES } & Very low* & 1.00 & 1.00 & 1.00 & 1.00 & 1.00 \\
\hline & Low & $\begin{array}{l}0.90(0.62- \\
1.29)\end{array}$ & $\begin{array}{l}0.92 \\
(0.68- \\
1.24)\end{array}$ & $\begin{array}{l}1.21 \\
(0.93- \\
1.57)\end{array}$ & $\begin{array}{l}0.80 \\
(0.61- \\
1.04)\end{array}$ & $\begin{array}{l}1.21 \\
(0.93- \\
1.57)\end{array}$ \\
\hline & Average & $\begin{array}{l}1.06(0.72- \\
1.56)\end{array}$ & $\begin{array}{l}0.85 \\
(0.62- \\
1.16)\end{array}$ & $\begin{array}{l}1.21 \\
(0.92- \\
1.58)\end{array}$ & $\begin{array}{l}0.85 \\
(0.65- \\
1.11)\end{array}$ & $\begin{array}{l}1.18 \\
(0.90- \\
1.54)\end{array}$ \\
\hline & High & $\begin{array}{l}0.96(0.65- \\
1.41)\end{array}$ & $\begin{array}{l}0.86 \\
(0.63- \\
1.17)\end{array}$ & $\begin{array}{l}1.09 \\
(0.83- \\
1.43)\end{array}$ & $\begin{array}{l}0.72 \\
(0.55- \\
0.95)^{\star \star}\end{array}$ & $\begin{array}{l}1.04 \\
(0.79- \\
1.36)\end{array}$ \\
\hline
\end{tabular}

Abbreviations: OR, odds ratio; $\mathrm{Cl}$, confidence interval; TG, triglyceride; $\mathrm{TC}$, total cholesterol; $\mathrm{HDL}$, highdensity lipoprotein; LDL, low-density lipoprotein; SES, socioeconomic status; BMI, body mass index; WHO, World Health Organization; WC, waist circumference; WHR, waist-to-hip ratio.

*Reference categories.

$\star \star$ Statistically significant $(P-v a l u e<0.05)$. 


\begin{tabular}{|c|c|c|c|c|c|c|}
\hline \multirow[t]{2}{*}{ Variable } & & Dyslipidemia & High TG & High TC & Low HDL & High LDL \\
\hline & Very high & $\begin{array}{l}0.97(0.64- \\
1.46)\end{array}$ & $\begin{array}{l}0.90 \\
(0.64- \\
1.27)\end{array}$ & $\begin{array}{l}1.16 \\
(0.87- \\
1.56)\end{array}$ & $\begin{array}{l}0.65 \\
(0.48- \\
0.87)^{\star \star}\end{array}$ & $\begin{array}{l}1.04 \\
(0.77- \\
1.39)\end{array}$ \\
\hline \multirow[t]{2}{*}{ Occupation } & Unemployed* & 1.00 & 1.00 & 1.00 & 1.00 & 1.00 \\
\hline & Employed & $\begin{array}{l}0.85(0.61- \\
1.20)\end{array}$ & $\begin{array}{l}0.95 \\
(0.69- \\
1.31)\end{array}$ & $\begin{array}{l}0.83 \\
(0.64- \\
1.07)\end{array}$ & $\begin{array}{l}0.94 \\
(0.72- \\
1.22)\end{array}$ & $\begin{array}{l}0.84 \\
(0.65- \\
1.09)\end{array}$ \\
\hline \multirow[t]{2}{*}{ Hookah } & No* & 1.00 & 1.00 & 1.00 & 1.00 & 1.00 \\
\hline & Yes & $\begin{array}{l}0.92(0.64- \\
1.33)\end{array}$ & $\begin{array}{l}0.81 \\
(0.60- \\
1.10)\end{array}$ & $\begin{array}{l}0.63 \\
(0.49- \\
0.82)^{\star \star}\end{array}$ & $\begin{array}{l}1.37 \\
(1.05- \\
1.77)^{\star \star}\end{array}$ & $\begin{array}{l}0.78 \\
(0.60- \\
1.01)\end{array}$ \\
\hline \multirow[t]{2}{*}{ Diabetes } & No* & 1.00 & 1.00 & 1.00 & 1.00 & 1.00 \\
\hline & Yes & $\begin{array}{l}1.23(0.86- \\
1.74)\end{array}$ & $\begin{array}{l}2.12 \\
(1.67- \\
2.69)^{\star \star}\end{array}$ & $\begin{array}{l}0.78 \\
(0.62- \\
0.98)^{\star \star}\end{array}$ & $\begin{array}{l}1.31 \\
(1.04- \\
1.65)^{\star \star}\end{array}$ & $\begin{array}{l}0.73 \\
(0.58- \\
0.92)^{\star \star}\end{array}$ \\
\hline \multirow[t]{2}{*}{ Hypertension } & No* & 1.00 & 1.00 & 1.00 & 1.00 & 1.00 \\
\hline & Yes & $\begin{array}{l}0.91(0.66- \\
1.24)\end{array}$ & $\begin{array}{l}1.35 \\
(1.06- \\
1.70)^{\star \star}\end{array}$ & $\begin{array}{l}1.20 \\
(0.96- \\
1.49)\end{array}$ & $\begin{array}{l}0.86 \\
(0.70- \\
1.07)\end{array}$ & $\begin{array}{l}1.07 \\
(0.87- \\
1.33)\end{array}$ \\
\hline \multirow[t]{3}{*}{ BMI } & $\begin{array}{l}\text { Normal \& } \\
\text { underweight* }\end{array}$ & 1.00 & 1.00 & 1.00 & 1.00 & 1.00 \\
\hline & Overweight & $\begin{array}{l}1.39(0.95- \\
2.03)\end{array}$ & $\begin{array}{l}1.59 \\
(1.13- \\
2.25)^{\star \star}\end{array}$ & $\begin{array}{l}1.23 \\
(0.93- \\
1.64)\end{array}$ & $\begin{array}{l}1.15 \\
(0.87- \\
1.52)\end{array}$ & $\begin{array}{l}1.10 \\
(0.83- \\
1.45)\end{array}$ \\
\hline & Obese & $\begin{array}{l}1.35(0.84- \\
2.18)\end{array}$ & $\begin{array}{l}1.65 \\
(1.10- \\
2.48)^{\star \star}\end{array}$ & $\begin{array}{l}1.29 \\
(0.91- \\
1.82)\end{array}$ & $\begin{array}{l}0.97 \\
(0.69- \\
1.37)\end{array}$ & $\begin{array}{l}1.07 \\
(0.76- \\
1.51)\end{array}$ \\
\hline \multirow[t]{2}{*}{ WHO WC } & Normal* & 1.00 & 1.00 & 1.00 & 1.00 & 1.00 \\
\hline & High & $\begin{array}{l}1.19(0.80- \\
1.77)\end{array}$ & $\begin{array}{l}1.09 \\
(0.74- \\
1.61)\end{array}$ & $\begin{array}{l}1.12 \\
(0.82- \\
1.51)\end{array}$ & $\begin{array}{l}1.19 \\
(0.88- \\
1.62)\end{array}$ & $\begin{array}{l}1.50 \\
(1.11- \\
2.03)^{\star \star}\end{array}$ \\
\hline Iranian WC & Normal* & 1.00 & 1.00 & 1.00 & 1.00 & 1.00 \\
\hline \multicolumn{7}{|c|}{$\begin{array}{l}\text { Abbreviations: OR, odds ratio; } \mathrm{Cl} \text {, confidence interval; TG, triglyceride; TC, total cholesterol; HDL, high- } \\
\text { density lipoprotein; LDL, low-density lipoprotein; SES, socioeconomic status; BMI, body mass index; } \\
\text { WHO, World Health Organization; WC, waist circumference; WHR, waist-to-hip ratio. }\end{array}$} \\
\hline \multicolumn{7}{|c|}{ *Reference categories. } \\
\hline \multicolumn{7}{|c|}{$\star \star$ Statistically significant $(P$-value $<0.05)$. } \\
\hline
\end{tabular}




\begin{tabular}{|c|c|c|c|c|c|c|}
\hline \multicolumn{2}{|l|}{ Variable } & \multirow{2}{*}{$\begin{array}{l}\text { Dyslipidemia } \\
0.96(0.66- \\
1.41)\end{array}$} & \multirow{2}{*}{$\begin{array}{l}\text { High TG } \\
1.21 \\
(0.89- \\
1.63)\end{array}$} & \multirow{2}{*}{$\begin{array}{l}\text { High TC } \\
0.80 \\
(0.62- \\
1.04)\end{array}$} & \multirow{2}{*}{$\begin{array}{l}\text { Low HDL } \\
1.18 \\
(0.91- \\
1.53)\end{array}$} & \multirow{2}{*}{$\begin{array}{l}\text { High LDL } \\
0.78 \\
(0.60- \\
1.02)\end{array}$} \\
\hline & High & & & & & \\
\hline \multirow[t]{2}{*}{ WHO WHR } & Normal* & 1.00 & 1.00 & 1.00 & 1.00 & 1.00 \\
\hline & High & $\begin{array}{l}2.48(1.75- \\
3.53)^{\star \star}\end{array}$ & $\begin{array}{l}3.01 \\
(1.87- \\
5.15)^{\star \star}\end{array}$ & $\begin{array}{l}1.52 \\
(1.12- \\
2.06)^{\star \star}\end{array}$ & $\begin{array}{l}1.72 \\
(1.27- \\
2.34)^{\star \star}\end{array}$ & $\begin{array}{l}1.61 \\
(1.18- \\
2.20)^{\star \star}\end{array}$ \\
\hline \multicolumn{7}{|c|}{$\begin{array}{l}\text { Abbreviations: OR, odds ratio; Cl, confidence interval; TG, triglyceride; TC, total cholesterol; HDL, high- } \\
\text { density lipoprotein; LDL, low-density lipoprotein; SES, socioeconomic status; BMl, body mass index; } \\
\text { WHO, World Health Organization; WC, waist circumference; WHR, waist-to-hip ratio. }\end{array}$} \\
\hline \multicolumn{7}{|c|}{ *Reference categories. } \\
\hline \multicolumn{7}{|c|}{$\star \star$ Statistically significant $(P$-value $<0.05)$. } \\
\hline
\end{tabular}


Table 4

Multiple regression analysis of predictors of lipid profile components

\begin{tabular}{|c|c|c|c|c|}
\hline Dependent variable & Predictors & B & P-value & Adjusted $\mathrm{R}^{2}$ \\
\hline \multirow[t]{12}{*}{ TG } & Age & 0.269 & 0.182 & \multirow{12}{*}{$\begin{array}{l}0.137 \\
(P \text {-value }<0.001)\end{array}$} \\
\hline & Years of education & 0.244 & 0.510 & \\
\hline & Weight & 1.113 & $<0.001$ & \\
\hline & WC & -2.905 & 0.127 & \\
\hline & $\mathrm{HC}$ & 0.623 & 0.737 & \\
\hline & WHR & 382.448 & 0.053 & \\
\hline & $\mathrm{BMI}$ & 2.511 & 0.002 & \\
\hline & FPG & 0.228 & $<0.001$ & \\
\hline & SBP & 0.416 & 0.003 & \\
\hline & DBP & -0.041 & 0.856 & \\
\hline & Daily calorie intake & 0.004 & 0.024 & \\
\hline & Weekly METs & -0.058 & 0.182 & \\
\hline \multirow[t]{12}{*}{ TC } & Age & 0.598 & $<0.001$ & \multirow{12}{*}{$\begin{array}{l}0.059 \\
(P-\text { value }<0.001)\end{array}$} \\
\hline & Years of education & 0.001 & 0.998 & \\
\hline & Weight & -0.425 & 0.039 & \\
\hline & WC & 0.083 & 0.946 & \\
\hline & $\mathrm{HC}$ & -0.135 & 0.910 & \\
\hline & WHR & -0.935 & 0.994 & \\
\hline & $\mathrm{BMI}$ & 1.564 & 0.003 & \\
\hline & FPG & 0.035 & 0.084 & \\
\hline & SBP & 0.079 & 0.381 & \\
\hline & DBP & 0.318 & 0.030 & \\
\hline & Daily calorie intake & 0.001 & 0.236 & \\
\hline & Weekly METs & 0.086 & 0.002 & \\
\hline
\end{tabular}

Abbreviations: TG, triglyceride; TC, total cholesterol; HDL, high-density lipoprotein; LDL, low-density lipoprotein; WC, waist circumference; HC, hip circumference; WHR, waist-to-hip ratio; BMI, body mass index; FPG, fasting plasma glucose; SBP, systolic blood pressure; DBP, diastolic blood pressure; MET, metabolic equivalent of task. 


\begin{tabular}{|c|c|c|c|c|}
\hline Dependent variable & Predictors & B & P-value & Adjusted $\mathrm{R}^{2}$ \\
\hline \multirow[t]{12}{*}{ HDL } & Age & 0.171 & $<0.001$ & \multirow{12}{*}{$\begin{array}{l}0.072 \\
(P-v a l u e<0.001)\end{array}$} \\
\hline & Years of education & 0.117 & 0.054 & \\
\hline & Weight & -0.300 & $<0.001$ & \\
\hline & WC & 1.294 & $<0.001$ & \\
\hline & $\mathrm{HC}$ & -0.883 & 0.004 & \\
\hline & WHR & -148.504 & $<0.001$ & \\
\hline & $\mathrm{BMI}$ & 0.022 & 0.870 & \\
\hline & FPG & 0.011 & 0.034 & \\
\hline & SBP & -0.013 & 0.559 & \\
\hline & DBP & 0.055 & 0.138 & \\
\hline & Daily calorie intake & $<0.001$ & 0.794 & \\
\hline & Weekly METs & 0.018 & 0.010 & \\
\hline \multirow[t]{12}{*}{ LDL } & Age & 0.358 & 0.001 & \multirow{12}{*}{$\begin{array}{l}0.031 \\
(P-v a l u e<0.001)\end{array}$} \\
\hline & Years of education & -0.179 & 0.379 & \\
\hline & Weight & -0.310 & 0.076 & \\
\hline & WC & -0.742 & 0.476 & \\
\hline & $\mathrm{HC}$ & 0.720 & 0.479 & \\
\hline & WHR & 83.109 & 0.442 & \\
\hline & $\mathrm{BMI}$ & 0.945 & 0.033 & \\
\hline & FPG & -0.014 & 0.414 & \\
\hline & SBP & 0.045 & 0.552 & \\
\hline & DBP & 0.230 & 0.064 & \\
\hline & Daily calorie intake & 0.001 & 0.480 & \\
\hline & Weekly METs & 0.080 & 0.001 & \\
\hline \multicolumn{5}{|c|}{$\begin{array}{l}\text { Abbreviations: TG, triglyceride; TC, total cholesterol; HDL, high-density lipoprotein; LDL, low-density } \\
\text { lipoprotein; WC, waist circumference; HC, hip circumference; WHR, waist-to-hip ratio; BMI, body mass } \\
\text { index; FPG, fasting plasma glucose; SBP, systolic blood pressure; DBP, diastolic blood pressure; MET } \\
\text { metabolic equivalent of task. }\end{array}$} \\
\hline
\end{tabular}

\section{Discussion}


The primary finding of the current study was the very high prevalence of dyslipidemia in women aged 35-70 years of the PERSIAN Bandare Kong Cohort Study, with $84.8 \%$ of the study population having at least one type of lipid abnormality based on ATP III criteria. High TC was the most common (53.9\%) followed by low HDL (50.8\%), high LDL (48\%), and high TG (26.2\%).

Prevalence of dyslipidemia in women was $87.7 \%$ in Najafipour et al.'s study (29), $61.3 \%$ in the study by Ebrahimi et al. (30), 85.1\% in Latifi et al.'s study (31), 80.4\% in a study on Turkish adults (32), and 37.6\% among Chinese women (2). In a systematic review and meta-analysis conducted by Tabatabei et al. on the prevalence of dyslipidemia in published articles until September 2011, hypercholesterolemia, low HDL, and high LDL were more prevalent in women compared to men (13). Esteghamati et al. showed that the prevalence of hypertriglyceridemia and hypercholesterolemia were $33.2 \%$ and $45.4 \%$ among females (33). The corresponding percentages were $41 \%$ and $23 \%$ in Japanese women aged 25-64 years (34), $25 \%$ and $37.2 \%$ in Turkish adults (35), $24.1 \%$ and $30.6 \%$ in Najafipour et al.'s study (29), and $65.1 \%$ and $47.5 \%$ in Latifi et al.'s study (31). The variety of the prevalence of dyslipidemia and its individual components across different studies can be explained by demographic, socioeconomic, and anthropometric features of study populations which will be discussed in details later on.

The results of this study showed that the prevalence of dyslipidemia steadily increased with age in women up to the age of 54, with an abrupt but slight decrease in the 55-59 age group, but it increased again thereafter probably due to hormonal changes during menopause. A similar trend was observed in Ebrahimi et al.'s study (30). Nonetheless, we found no significant increase in the odds of dyslipidemia with age based on the logistic regression analysis. Yet, other studies in different parts of the world have shown that the risk of different types of dyslipidemia increases with age in both men and women (3537).

We found no correlation between marital status and dyslipidemia or any of its components, which was in line with the findings of Ebrahimi et al. (30). Whereas, aside from low HDL, other lipid abnormalities were higher in married and widowed individuals compared to singles in the study by Erem et al. (35).

According to the findings of the current study, having a high school diploma was protective against dyslipidemia; however, there was no significant association between the level of education and individual components of dyslipidemia. Quite similarly, dyslipidemia was not influenced by level of education in a study conducted in India (10). In addition, this was partially consistent with the results of Ebrahimi et al.'s study, in which no relationship was found between the risk of developing different types of dyslipidemia and the level of education (30). On the contrary, Erem et al. in their study to estimate the prevalence of dyslipidemia and associated factors among Turkish adults, demonstrated that the risk of dyslipidemia was higher in those with lower education level. They justified their findings by higher exposure to risk factors such as poor eating habits and working conditions, difficulty to access health services, and stress in those with low level of education (35). Of note, the findings of the above-mentioned studies were with respect to both men and women. Results can be different when only women are concerned. 
We found that women living in rural areas were at significant decreased risk of developing dyslipidemia, low HDL, and high LDL. The odds of hypertriglyceridemia and hypercholesterolemia, although insignificant, was also lower in women living in rural areas. Conversely, Cui et al. demonstrated that the prevalence of hypercholesterolemia was higher in rural areas compared to urban areas for women (34). Similarly Tripathy et al. demonstrated that living in rural areas increased the risk of dyslipidemia and high TC (38). The reason for these inconsistencies may be the consumption of fat-rich foods such as high-fat dairy by individuals living in rural areas in the two studies.

Over the past decade, there has been a decline in consumption of traditional foods, while use of high-fat, high-calorie, low-fiber, and processed foods has increased. This unhealthy diet together with insufficient physical activity, are risk factors for obesity and hypertriglyceridemia (39-41). Among the participants of the current study, $39 \%$ were overweight and $30.5 \%$ were obese. Overweight and obesity were only significantly correlated with hypertriglyceridemia but not dyslipidemia or other lipid abnormalities. Among the anthropometric indices, high WHR was the best predictor of dyslipidemia and all of its components. Obesity has been reported to be associated with hypertriglyceridemia in many studies $(9,10,35-37,42$, 43). It should be noted that contrary to our findings, obesity has been proposed as a risk for hypercholesterolemia in some studies $(9,10,35,42)$.

The odds of hypertriglyceridemia was significantly higher in women with hypertension in our study, while neither dyslipidemia nor any other components were associated with hypertension. This was in agreement with the findings of Ebrahimi et al.; however, they also reported a significant correlation between high blood pressure and dyslipidemia (30). Tabrizi et al. reported similar results (44). Despite comparable findings regarding the association between hypertension and hypertriglyceridemia in a study in India, dyslipidemia and other lipid abnormalities were also significantly correlated in this study (38).

One limitation of the current study was that although, lipid-lowering medications were taken into account, some women with diabetes may have failed to indicate that they were taking these agents, which resulted in contradictory findings regarding the relationship between dyslipidemia or its components with diabetes. Another limitation was the assessment of physical activity which was reported in METs. The positive effect of physical activity on serum lipids, plasma glucose, and many other CVD risk factors has been previously established; therefore, the increase in TC and LDL with higher weekly METs observed in the current study can in part be due to inaccurate evaluation of physical activity. One more limitation was the daily calorie intake that was not subdivided based on specific foods. Calorie content of fat-rich foods would have been more valuable in the assessment of the correlation between lipid abnormalities and daily calorie intake.

\section{Conclusions}

Dyslipidemia was highly prevalent in women of the PERSIAN Bandare Kong Cohort Study. High TC was the most common, and high TG the least common lipid abnormalities in this population. High WHR put women aged 35-70 years at high risk of dyslipidemia and all of its components and appears to be the 
best predictive anthropometric index with regard to lipid abnormalities. Women aged 50-54 years were at highest risk of developing lipid abnormalities compared to other age groups. Living in rural areas positively influenced the lipid profile of women, while marital status and employment had no effect on it. Except for the positive effect of having a high school diploma on dyslipidemia, level of education did not affect lipid abnormalities. Although the odds of high TG was higher in those with hypertension. The significance of hookah use and diabetes for lipid abnormalities was paradoxical. These findings should be taken into consideration in the preparation of future management and prevention guidelines designated for this specific population.

\section{Declarations}

\section{Abbreviations}

- ADA

- American Diabetes Association

- ATP III

- Adult Treatment Panel III

- BMI

- body mass index

- BP

- blood pressure

- $\mathrm{Cl}$

- confidence interval

- CVD

- cardiovascular disease

- DALYS

- disability-adjusted life years

- DBP

- diastolic blood pressure

- FPG

- fasting plasma glucose

- $\mathrm{HC}$

- hip circumference

- HDL

- high-density lipoprotein

- LDL

- low-density lipoprotein

- MET

- metabolic equivalent of task

- OR

- odds ratio 
- PERSIAN

- Prospective Epidemiological Research Studies in IrAN

- SBP

- systolic blood pressure

- SES

- socioeconomic status

- SPSS

- Statistical Package for the Social Sciences

- TC

- total cholesterol

- TG

- triglyceride

-WC

- waist circumference

- WHO

- World Health Organization

- WHR

- waist-to-hip ratio

\section{Declarations}

\section{Ethics approval and consent to participate}

The cohort study was given ethical approval by the Ethics Committee of Hormozgan University of Medical Sciences.

\section{Availability of data and materials}

The datasets used and/or analyzed during the current study are available from the corresponding author on reasonable request.

\section{Competing interests}

The authors declare that they have no competing interests.

\section{Funding}

The Iranian Ministry of Health and Medical Education has contributed to the funding used in the PERSIAN Cohort through grant number 700/534.

\section{Author's contributions}


MN designed the study and was a major contributor in writing the manuscript. MK was consulted on the possible associated factors to be taken into account and wrote the manuscript. HY performed the statistical analysis. MK interpreted the analyzed data. All authors read and approved the final manuscript.

\section{Acknowledgments}

BKNCD is a part of PERSIAN national cohort and we would like to thank Professor Reza Malekzadeh, Deputy of Research and Technology at the Ministry of Health and Medical Education of Iran and Director of the PERSIAN cohort, and also Dr. Hossein Poustchi, Executive Director of the PERSIAN cohort, for all their supports during design and running of BKNCD. The Iranian Ministry of Health and Medical Education has contributed to the funding used in the PERSIAN Cohort through grant number 700/534.

\section{References}

1. Stone NJ, Robinson JG, Lichtenstein AH, Merz CNB, Blum CB, Eckel RH, et al. 2013 ACC/AHA guideline on the treatment of blood cholesterol to reduce atherosclerotic cardiovascular risk in adults: a report of the American College of Cardiology/American Heart Association Task Force on Practice Guidelines. Journal of the American College of Cardiology. 2014;63(25 Part B):2889 - 934.

2. Qi L, Ding X, Tang W, Li Q, Mao D, Wang Y. Prevalence and risk factors associated with dyslipidemia in Chongqing, China. Int J Environ Res Public Health. 2015;12(10):13455-65.

3. Mohammadbeigi A, Moshiri E, Mohammadsalehi N, Ansari H, Ahmadi A. Dyslipidemia prevalence in Iranian adult men: the impact of population-based screening on the detection of undiagnosed patients. The world journal of men's health. 2015;33(3):167-73.

4. Stevens W, Peneva D, Li JZ, Liu LZ, Liu G, Gao R, et al. Estimating the future burden of cardiovascular disease and the value of lipid and blood pressure control therapies in China. BMC Health Serv Res. 2016;16(1):175.

5. World Health O. Global status report on noncommunicable diseases 2014: World Health Organization; 2014.

6. Asgari F, Mirzazadeh A, Heidaria H. Iran-non-communicable diseases risk factors surveillance-Data book for 2007. Tehran: Chakameh Ava Group. 2009:1-82.

7. Hatmi ZN, Tahvildari S, Motlag AG, Kashani AS. Prevalence of coronary artery disease risk factors in Iran: a population based survey. BMC Cardiovasc Disord. 2007;7(1):32.

8. Sharifi F, Mousavinasab SN, Soruri R, Saeini M, Dinmohammadi M. High prevalence of low highdensity lipoprotein cholesterol concentrations and other dyslipidemic phenotypes in an Iranian population. Metabolic Syndrome Related Disorders. 2008;6(3):187-95.

9. Yarahmadi SH, Etemad K, Hazaveh ARM, Azhang N. Urbanization and non-communicable risk factors in the capital city of 6 big provinces of Iran. Iranian journal of public health. 2013;42(Supple1):113. 
10. Guptha S, Gupta R, Deedwania P, Bhansali A, Maheshwari A, Gupta A, et al. Cholesterol lipoproteins and prevalence of dyslipidemias in urban Asian Indians: a cross sectional study. indian heart journal. 2014;66(3):280-8.

11. Aguilar-Salinas CA, Gómez-Pérez FJ, Rull J, Villalpando S, Barquera S, Rojas R. Prevalence of dyslipidemias in the Mexican national health and nutrition survey 2006. Salud publica de Mexico. 2010;52:44-53.

12. Azizi F, Rahmani M, Ghanbarian A, Emami H, Salehi P, Mirmiran P, et al. Serum lipid levels in an Iranian adults population: Tehran Lipid and Glucose Study. Eur J Epidemiol. 2003;18(4):311-9.

13. Tabatabaei-Malazy O, Qorbani M, Samavat T, Sharifi F, Larijani B, Fakhrzadeh H. Prevalence of dyslipidemia in Iran: a systematic review and meta-analysis study. International journal of preventive medicine. 2014;5(4):373.

14. Han X, Fox DS, Chu M, Dougherty JS, McCombs J. Primary Prevention Using Cholesterol-Lowering Medications in Patients Meeting New Treatment Guidelines: A Retrospective Cohort Analysis. Journal of managed care specialty pharmacy. 2018;24(11):1078-85.

15. Heart Protection Study Collaborative G. Heart Protection Study Collaborative Group MRC/BHF heart protection study of cholesterol lowering with simvastatin in, 20, 536 high-risk individuals: a: randomised placebo-controlled trial. Lancet. 2002;360:7-22.

16. Grundy SM, Cleeman JI, Merz CNB, Brewer HB, Clark LT, Hunninghake DB, et al. Implications of recent clinical trials for the national cholesterol education program adult treatment panel III guidelines. $\mathrm{J}$ Am Coll Cardiol. 2004;44(3):720-32.

17. Jayarama N, Reddy M, Lakshmaiah V. Prevalence and pattern of dyslipidemia in type 2 diabetes mellitus patients in a rural tertiary care centre, southern India. Glob J Med Public Health. 2012;1:248.

18. Snehalatha C, Nanditha A, Shetty AS, Ramachandran A. Hypertriglyceridaemia either in isolation or in combination with abdominal obesity is strongly associated with atherogenic dyslipidaemia in Asian Indians. Diabetes Res Clin Pract. 2011;94(1):140-5.

19. Djelilovic-Vranic J, Alajbegovic A, Zelija-Asimi V, Niksic M, Tiric-Campara M, Salcic S, et al. Predilection role diabetes mellitus and dyslipidemia in the onset of ischemic stroke. Medical Archives. 2013;67(2):120.

20. William B, Riaz I, Mubashir S, Aslam K, Saeed A, Anjum I. DYSLIPIDEMIA AS A RISK, FACTOR FOR DEVELOPING HYPERTENSION AND CARDIOVASCULAR DISEASE IN FEMALES OF REPRODUCTIVE AGE. Journal of Natural Applied Sciences Pakistan. 2019;1(2):139-47.

21. Grundy SM. George Lyman Duff Memorial Lecture. Multifactorial etiology of hypercholesterolemia. Implications for prevention of coronary heart disease. Arteriosclerosis thrombosis: a journal of vascular biology. 1991;11(6):1619-35.

22. Poustchi H, Eghtesad S, Kamangar F, Etemadi A, Keshtkar A-A, Hekmatdoost A, et al. Prospective epidemiological research studies in Iran (the PERSIAN Cohort Study): rationale, objectives, and design. Am J Epidemiol. 2018;187(4):647-55. 
23. World Health $\mathrm{O}$. Waist circumference and waist-hip ratio: report of a WHO expert consultation, Geneva, 8-11 December 2008. 2011.

24. Azizi F, Khalili D, Aghajani H, Esteghamati AR, Hosseinpanah F, Delavari AR, et al. Appropriate waist circumference cut-off points among Iranian adults: the first report of the Iranian National Committee of Obesity. 2010.

25. World Health $\mathrm{O}$. Report of a WHO consultation on obesity. Obesity: preventing and managing the global epidemic Geneva: World Health Organization. 1998.

26. James PA, Oparil S, Carter BL, Cushman WC, Dennison-Himmelfarb C, Handler J, et al. 2014 evidence-based guideline for the management of high blood pressure in adults: report from the panel members appointed to the Eighth Joint National Committee (JNC 8). Jama. 2014;311(5):507-20.

27. Riddle MC, Bakris G, Blonde L, Boulton AJM, D'alessio D, De Groot M, et al. Introduction: standards of medical care in diabetes-2018. Diabetes Care. 2018;41(Supplement 1):1-2.

28. Grundy SM, Cleeman JI, Daniels SR, Donato KA, Eckel RH, Franklin BA, et al. Diagnosis and management of the metabolic syndrome: an American Heart Association/National Heart, Lung, and Blood Institute scientific statement. Circulation. 2005;112(17):2735-52.

29. Najafipour H, Shokoohi M, Yousefzadeh G, Azimzadeh BS, Kashanian GM, Bagheri MM, et al. Prevalence of dyslipidemia and its association with other coronary artery disease risk factors among urban population in Southeast of Iran: results of the Kerman coronary artery disease risk factors study (KERCADRS). Journal of Diabetes Metabolic Disorders. 2016;15(1):49.

30. Ebrahimi H, Emamian MH, Hashemi H, Fotouhi A. Dyslipidemia and its risk factors among urban middle-aged Iranians: A population-based study. Diabetes Metabolic Syndrome: Clinical Research Reviews. 2016;10(3):149-56.

31. Latifi SM, Moradi L, Shahbazian H, Aleali AM. A study of the prevalence of dyslipidemia among the adult population of Ahvaz, Iran. Diabetes \& Metabolic Syndrome: Clinical Research \& Reviews. 2016;10(4):190-3.

32. Bayram F, Kocer D, Gundogan K, Kaya A, Demir O, Coskun R, et al. Prevalence of dyslipidemia and associated risk factors in Turkish adults. J Clin Lipidol. 2014;8(2):206-16.

33. Esteghamati A, Meysamie A, Khalilzadeh O, Rashidi A, Haghazali M, Asgari F, et al. Third national Surveillance of Risk Factors of Non-Communicable Diseases (SuRFNCD-2007) in Iran: methods and results on prevalence of diabetes, hypertension, obesity, central obesity, and dyslipidemia. BMC Public Health. 2009;9(1):167.

34. Cui M, Chiang C, Cui R, Honjo K, Yatsuya H, Watson BM, et al. Prevalence and correlates of dyslipidemia among men and women in Palau: Findings of the Palau STEPS survey 2011-2013. J Epidemiol. 2018:JE20170127.

35. Erem C, Hacihasanoglu A, Deger O, Kocak M, Topbas M. Prevalence of dyslipidemia and associated risk factors among Turkish adults: Trabzon lipid study. Endocrine. 2008;34(1-3):36-51.

36. Khader YS, Batieha A, El-Khateeb M, Al Omari M, Ajlouni K. Prevalence of dyslipidemia and its associated factors among Jordanian adults. Journal of Clinical Lipidology. 2010;4(1):53-8. 
37. Crawford AG, Cote C, Couto J, Daskiran M, Gunnarsson C, Haas K, et al. Prevalence of obesity, type II diabetes mellitus, hyperlipidemia, and hypertension in the United States: findings from the GE Centricity Electronic Medical Record database. Population health management. 2010;13(3):151-61.

38. Tripathy JP, Thakur JS, Jeet G, Chawla S, Jain S, Pal A, et al. Burden and risk factors of dyslipidemiaresults from a STEPS survey in Punjab India. Diabetes \& Metabolic Syndrome: Clinical Research \& Reviews. 2017;11:S21-S7.

39. World Health O. Facts on obesity. Retrieved February.1:2016.

40. Mozaffarian D. Dietary and policy priorities for cardiovascular disease, diabetes, and obesity: a comprehensive review. Circulation. 2016;133(2):187-225.

41. Hata Y, Nakajima K. Life-style and serum lipids and lipoproteins. J Atheroscler Thromb. 2000;7(4):177-97.

42. Deng $B$, Luo T, Huang Y, Shen T, Ma J. Prevalence and determinants of hyperlipidemia in moderate altitude areas of the Yunnan-Kweichow plateau in Southwestern China. High Alt Med Biol. 2012;13(1):13-21.

43. Montano GT, Witherell R, Mar A, Szpunar SM, Anne P. Predictors of screening for hyperlipidemia in an urban pediatric tertiary care center. Clin Pediatr. 2015;54(3):244-8.

44. Tabrizi JS, Nikniaz L, Sadeghi-Bazargani H, Farahbakhsh M, Nikniaz Z, Farhangi MA, et al. Prevalence of Dyslipidemia in Urban and Rural Areas of the Northwest of Iran: The Sociodemographic, Dietary and Psychological Determinants. Iranian journal of public health. 2019;48(5):925. 